\title{
Interdisciplinariedad en la formación universitaria del diseño gráfico: entre la teoría y la práctica
}

\author{
ARODI MORALES HOLGUÍN* \\ EDGAR GONZÁLEZ BELLO ${ }^{* *}$
}

Universidad de Sonora - México

Recibido el 30-01-20; primera evaluación el 19-12-20; segunda evaluación el 14-01-21; aceptado el 23-01-21

\section{Resumen}

Las tendencias en la formación universitaria del diseño destacan el aporte de otras disciplinas como promotores de conocimientos indispensables para el ejercicio profesional. Específicamente, el diseño gráfico se ubica como un campo que demanda una base formativa interdisciplinar acorde con su dinámica de acción. Mediante la aplicación de un cuestionario a 126 profesores universitarios de México, se analiza la pertinencia del diseño gráfico para vincularse con otras disciplinas, en correspondencia con acciones docentes que se llevan a cabo. Los resultados advierten una disyuntiva entre la trascendencia concedida a la interdisciplinariedad frente al escaso compromiso que se manifiesta en la formación, diferenciada por tipos de universidad. Se concluye la pertinencia de replantear las propuestas de formación y esencialmente promover una enseñanza que trascienda los confines de la misma disciplina.

Palabras clave: diseño gráfico, interdisciplinariedad, educación universitaria, formación profesional, método de diseño.

\footnotetext{
* Profesor-investigador de tiempo completo del Departamento de Arquitectura y Diseńo en la Universidad de Sonora. Doctor en Arquitectura Diseño y Urbanismo, Maestría en Administración, máster en Publicidad y Marketing, y licenciatura en Diseńo Gráfico. Es reconocido por el Sistema Nacional de Investigadores (SNI). Es editor responsable de la revista MADGU de la Univ. de Sonora. Es evaluador externo de programas de posgrado y dictaminador de diversas revistas. Ha dirigido un despacho de diseńo por más de 15 años. Correo electrónico: redeshmo@gmail.com. https://orcid.org/0000-0001-9241-032X ** Profesor-investigador de tiempo completo del Departamento de Psicología y Ciencias de la Comunicación en la Universidad de Sonora. Doctor en Ciencias Sociales, Maestría en Innovación Educativa. Es reconocido por el Sistema Nacional de Investigadores (SNI). Es miembro asociado del Consejo Mexicano de Investigación Educativa (COMIE) en México. Es evaluador externo del programa nacional CERTIDEMS de la ANUIES, además de participar como dictaminador de diversas revistas y programas de becas del CONACYT. Correo electrónico: edgar.gonzalezb@gmail. com. https://orcid.org/0000-0001-6297-2516
} 


\section{Interdisciplinarity in university education of graphic design: between theory and practice}

\section{Abstract}

The tendencies in the university education of design, emphasize the contribution of other disciplines as a promoter of indispensable knowledge for professional practice. Specifically, graphic design is located as a field that demands an interdisciplinary training base according to its dynamics of action. Through the application of a questionnaire to 126 university professors in Mexico, the relevance of graphic design to link up with other disciplines is analyzed, in correspondence with teaching actions carried out. The results show a dilemma between the importance granted to interdisciplinarity versus the low level of commitment manifested in training, differentiated by types of university. It concludes with the relevance of rethinking the training proposals and essentially promoting education that transcends the confines of the same discipline.

Keywords: Graphic design, interdisciplinarity, university education, professional training, design method.

\section{Interdisciplinaridade no ensino universitário de design gráfico: entre teoria e prática}

\section{RESUMO}

As tendências na formação universitária do design enfatizam a contribuição de outras disciplinas como promotoras de conhecimentos indispensáveis para o exercício profissional. Especificamente, o design gráfico está localizado como um campo que exige uma base de treinamento interdisciplinar de acordo com sua dinâmica de ação. Através da aplicação de um questionário a 126 professores universitários do México, é analisada a relevância do design gráfico para ligação com outras disciplinas, em correspondência com as açôes de ensino realizadas. Os resultados alertam para um dilema entre a importância conferida à interdisciplinaridade diante do baixo comprometimento que se manifesta na formação, diferenciada por tipos de universidade. Conclui com a relevância de repensar as propostas de treinamento e, essencialmente, promover um ensino que transcenda os limites da mesma disciplina.

Palavras-chave: design gráfico, interdisciplinaridade, educação universitária, formação profissional, Método de design. 


\section{INTRODUCCIÓN}

En todo el mundo, dentro del escenario de la formación universitaria, se ha buscado continuamente la colaboración entre disciplinas que, de vez en cuando, operen simultáneamente como valor agregado para la formación, pues tienden a convertirse en casi una necesidad para la práctica de las profesiones, incluyendo aquellas como el diseño (Kirci, 2016). Específicamente en el contexto del diseño gráfico, contenidos y métodos que interactúan, conciben a la interdisciplinariedad más allá de una forma de organizar contenidos y se convierte en una metodología que se revierte y tiene influencias en los contenidos mismos que la proponen.

La interdisciplinariedad ha tenido lugar en la búsqueda de soluciones a los problemas complejos que rebasan a las disciplinas en sí, siendo necesario la integración de conceptos como paradigmas multidisciplinares (Vázquez y Manassero, 2017). Además, debe ser concebida no solo como un lugar de conjunción de las disciplinas, y que por separado ofrecen solo una visión técnica de su propia especialidad (Carvajal, 2010), sino como un puntogénesis de la creación donde se dan las condiciones para la emergencia de nuevos e híbridos conocimientos. Con base en esto, Roncallo-Dow (2013) señala que la consideración de la interdisciplinariedad se ha convertido en una necesidad debido a que la especialización absoluta cae en el anacronismo. Esto también supone que existen distintas concepciones de interdisciplinariedad y se vinculan con las especificidades del escenario en que son utilizadas (Carvajal, 2010).

En esa misma línea, Rivera (2013) entiende que el diseñador va evolucionando a tal grado de requerir ciertas habilidades — razonamiento, análisis, investigación, etc. - con el propósito de favorecer la obtención de mejores resultados; determinantes en la transición del diseñador reproductor a un perfil más innovador y de vanguardia, para lo cual la interdisciplinariedad emerge como requisito elemental, pues la innovación hoy requiere perspectivas de pensamiento y de análisis distintas a las tradicionales, que trasciendan al diseño interconectándose con otras disciplinas, donde, como argumenta Calvache (2017), los problemas de diseño y su planteamiento son cada vez más complejos y la adopción de una visión acorde a la complejidad es imperativa de cara a un ejercicio innovador, alejada de las prácticas tradicionales.

De esta manera, el diseñador poseedor de un pensamiento interdisciplinar requerirá la adopción de una visión horizontal, opuesta a una que ha sido imperante en la cultura del diseño, más de corte vertical, a través de la cual sea posible la integración e interacción participativa que promueva el 
diálogo circular y por ende, el enriquecimiento de sus criterios. Aspectos que en la formación parecen no ser abordados con la relevancia que convendría de acuerdo con la realidad y demandas que el entorno manifiesta; carencias que impactan en el diseñador y su ejercicio profesional.

La adopción de la perspectiva interdisciplinar podría diluir la rigidez presente en el pensamiento del diseńador que tiende a no legitimar las distintas disciplinas y no asumirlas como partícipes del diseño. Ante ello, el presente artículo busca analizar la pertinencia del diseńo gráfico para vincularse con otras disciplinas, en correspondencia con acciones docentes que se llevan a cabo.

Desde una perspectiva integral, Hayawi y Kamil (2015) asumen el diseño gráfico como un componente que desempeña una función interdisciplinaria para servir como vínculo entre el conocimiento y las habilidades profesionales, psicológicas y pedagógicas; también como una forma universal para comprender el entorno desde la perspectiva de la razonabilidad estética y funcional.

\section{MARCO DE REFERENCIA}

En el contexto de la sociedad actual, el diseño, independiente a su campo de aplicación —arquitectónico, gráfico, industrial, interiores, moda- cuya presencia se ha vuelto cotidiana, puede ser considerado como una actividad interdisciplinaria que está íntimamente relacionada con el diario vivir, pues prácticamente con todo lo que se interactúa es resultado del diseño. Por tanto, Mose, Dalsgaard y Halskov (2017) proponen concebir el diseño como una acción creativa que, de manera incremental o disruptiva, busca adaptar y/o mejorar lo existente mediante aportaciones novedosas. Esta contribución requiere de un fin práctico que cumpla con ciertos requisitos: ser funcional, significativo y concreto, además de contar con un componente social, como también estar íntimamente asociado con la industria, y asumido principalmente desde la cultura de la industria (Pimentel, 2010). Entonces, el diseño se convierte en una práctica donde se forjan y determinan ideas, así como formas que posteriormente se materializan (Sánchez, 2016).

Desde otra perspectiva, el diseño se concibe como la creación de la forma que es capaz de establecer valores culturales, abarcando esferas socioculturales, económicas y políticas (García-Cordero y Buitrago-López, 2017). Entonces, el saber y hacer de un diseñador implica potencialmente aprender a ser creativo, original, innovador, ingeniosos, a través del uso de diferentes elementos como: inteligencia, cognición, intuición y experiencia (Rodríguez y Montiel, 2020), 
así también demostrar la capacidad para recibir, asumir e integrar los aportes que provienen de otras disciplinas. Se trata de un elemento que va más allá de la intuición o la creatividad innata, pues indefectiblemente requiere de conciencia y que desde el currículo se enfoquen esfuerzos que permitan la práctica interdisciplinar.

De forma específica, el diseño gráfico es un área que se ha sostenido desde una base comunicativa-visual en constante evolución, impulsada por las tecnologías y la vigente cultura del consumo mediante el uso estratégico de mensajes visuales, los cuales son dirigidos a mercados definidos con propósitos casi siempre comerciales. Productos gráficos que, en la constante búsqueda innovadora, demandan al diseñador manifestar la habilidad de construir productos novedosos; responsabilidad que indefectiblemente requieren seguir una estructura interdisciplinar.

Entonces, ofrecer ese tipo de formación redirige ciertas exigencias hacia la enseñanza del diseño al incorporar una mayor complejidad, en parte por la influencia de los avances tecnológicos, la evolución cultural, en conjunto con nuevos materiales y procesos, potenciando un grado mayor de interdisciplinariedad dentro de su dinámica y actividad proyectual, los cuales demandan razonamientos propios en la conceptualización y los planteamientos estructurales (Tresserras, 2015), donde igualmente los nuevos conocimientos propios de otras disciplinas cercanas logren promoverlo. En este sentido, la aparición del diseño integrador basado en una amplia gama de disciplinas ha impulsado la discusión y la exploración entre varios participantes.

Con relación a las formaciones en diseño, Tresserras (2015) subraya que dentro del contexto de las universidades, hay que reconsiderar los planteamientos pedagógicos ante el mayor protagonismo de la interdisciplinariedad, lo que ha planteado el reto de redefinir los planes de estudios en el cual las asignaturas de proyectos sean los ejes vertebradores de la enseñanza, y las asignaturas se interrelacionen con la propuesta formativa, sumando y entrelazando conocimientos, metodologías, técnicas y herramientas, promoviendo así una mayor definición y efectividad en el resultado final, donde la interdisciplinariedad se destaca como la tendencia que enmarca la dinámica educativa (Lyall y Meagher, 2012).

Particularmente en México, se ha denotado cómo la formación universitaria que se propone en el ámbito del diseño gráfico ha buscado evolucionar al mismo ritmo que otras disciplinas, tanto en su enseñanza como en la práctica. A pesar de estos esfuerzos, el estudio de Sotelo (2014) advierte varias inconsistencias: remarca que no existe una tendencia definida en cuanto a formas de enseñanza y el currículo de esta profesión, dado que cada universidad 
establece su perfil de egreso según las necesidades laborales y económicas del contexto propio.

Estas exigencias, señala, han promovido planes de estudios disímiles que pueden ser diferenciados en tres categorías: aquellos enfocados en las habilidades generales propias de las áreas de diseño; otros que impulsan una especialización en la etapa final con perfil esencialmente directivo-empresarial; y los que buscan formar a profesionistas con un perfil más técnico pero requerido por la manufactura. A esto se suma la situación de profesores, una parte de los cuales carece de conocimientos pedagógicos y didácticos, otros que nunca han ejercido la disciplina, mientras otra parte accede a la docencia debido a que se encuentran desempleados.

En consecuencia, se puede suponer que la enseñanza del diseño ha establecido un estilo fundamentado en principios teóricos, donde prevalecen aquellos que datan de hace cien años en la Bauhaus. Formación cimentada en muchos casos en una base anacrónica, basada en estructuras causales de pensamiento y práctica (Morín, 2004), consideradas insuficientes para las condiciones y necesidades que actualmente destacan. El contexto del diseño del siglo XXI exige que los equipos se compongan por consultores multidisciplinarios en red que trabajen individualmente y como parte de un equipo diverso donde la comunicación y la colaboración entre los miembros sea no solo importante sino inevitable; contexto que exige convergencias significativas entre herramientas digitales, procesos subyacentes y comportamientos de los usuarios (D’souza, 2016); trascendiendo la concepción tradicional del diseño de perfil unidireccional.

Como resultado, en muchas universidades de México como en otras partes del mundo, la formación en diseño sigue una enseñanza basada en el sistema de talleres, método que podría seguir siendo pertinente como base didáctica, siempre y cuando ofrezca los resultados que son indispensables para el desarrollo de herramientas cognitivas enfocadas en identificar y analizar situaciones que son oportunidades para este campo (Rivera, 2018), además de una necesaria cooperación de múltiples disciplinas del conocimiento (Chou y Wong, 2015). No obstante, esto a veces es algo que poco acontece, a tal grado que Pimentel (2010) advierte como los «nuevos» diseñadores continúan formándose con planes obsoletos, junto a una protagonista acción docente.

Esta situación permite dilucidar cómo la enseñanza del diseño en México evidencia deficiencias, retos y necesidades, pues centra su formación principalmente en contenidos técnicos y conceptuales, y en menor proporción en el desarrollo de herramientas cognitivas descritas, donde asumir la realidad compleja del entorno que envuelve al diseño a través de una visión 
interdisciplinar es algo pertinente. En ese sentido, el estudio de Cepeda y Carrillo (2019) identificó competencias y habilidades que el entorno laboral mexicano demanda para la obtención de un empleo en los diseñadores; muchas de las cuales no forman parte del modelo que sigue la formación universitaria, lo que limitan en muchos sentidos dichas oportunidades.

Esto se traduce en profesionistas que carecen de una formación integral, pues suelen exhibir habilidades marcadas como ciertas insuficiencias en el ejercicio profesional, afectando su adecuada inclusión en el mercado laboral; exhibiendo algunos la falta de una visión íntegra y real de los problemas, lo que impide llegar a comprenderlos cabalmente, conduciéndolos a propuestas ciegas que se traducen en la práctica en soluciones inadecuadas para los problemas que pretenden resolver.

Pese a todas estas adversidades, Dorochesi (2010) concibe cómo un profesional del diseño debe ser orientado a la construcción de un agente articulador de la creatividad y la innovación en las empresas, lo que demanda desarrollar competencias que le faculten para visualizar y operar con nuevas coordenadas en el espacio laboral, incluida la habilidad para asimilar la dilución de las tradicionales fronteras disciplinares con respecto al diseño, requiriéndose el promocionar una formación acorde a una visión diferente que promueva una accesible y coherente vinculación con otras disciplinas, favoreciendo así mejores soluciones. Se trata de un diseñador capaz de desenvolverse desde múltiples perspectivas, con visión innovadora y dispuesto a arriesgar; consciente de las ventajas de adoptar la interdisciplinariedad en la lógica como en la práctica.

Entonces, se reconoce una perspectiva que en muchos casos no parece orientar de mejor manera a la formación del diseño gráfico en las universidades de México. Así, la interdisciplinariedad surge como un elemento que propone una mejor preparación profesional, al constituirse como el medio para lograr una visión más integral y transversal (Brandão et al., 2014). De esta manera, el diseńo interactúa de manera cercana con disciplinas como el marketing, psicología, economía, management, neurociencias, tecnología, interconectándose también con otras, de acuerdo con el tipo de problema a desarrollar. No obstante, dicha vocación interdisciplinar resulta escasa en el ámbito académico.

En el contexto universitario de México, anteriormente Soto (1993) señaló que, en función de las necesidades de formación, se ha vuelto pertinente retomar un modelo flexible que posibilite el diseño y la reestructuración de planes de estudio en las universidades públicas, donde se aborde la formación interdisciplinaria de acuerdo con las necesidades contextuales, además de estrechar el vínculo universidad-sociedad, algo que se ha promovido e integrado en 
una amplia variedad de currículos universitarios. Sin embargo, en la mayoría de los programas formativos en diseño gráfico, se ha establecido una serie de materias organizadas por ejes que atomizan el conocimiento y aunque se tiene la asignatura de proyectos o taller para aglutinar el conocimiento adquirido, se carecen de mecanismos para reunir, utilizar y evaluar los saberes que son útiles para resolver problemas complejos (Herrera y Neve, 2011).

En ese sentido, desde el pensamiento del diseño interdisciplinario, Ambrose (2017) señala que los problemas requieren de una reflexión y colaboración creativa e interdisciplinaria para permitir que las personas y los equipos remedien el dogmatismo que dificulta la percepción de la complejidad y que se constituye como un obstáculo para el trabajo creativo e innovador.

Como resultado, los programas de diseño deben contar con asignaturas donde los estudiantes se relacionen, tomen decisiones y resuelvan problemas complejos con el uso de fuentes interdisciplinarias. Se tratará entonces de la exigencia de un trabajo que demanda un análisis integral de los fenómenos y procesos, que no pueden ser explicados de manera parcial desde perspectivas disciplinarias (Rivera, Higuera, Rubio, Utrilla y Santamaría, 2014).

Similar a la situación de México es la de Chile, donde Donoso (2016) muestra que existe una dispersión en la formación de diseñadores, en la cual los programas de estudios tienen baja compatibilidad entre sí, dados los distintos perfiles de sus programas, desde un enfoque empresarial, artesanado, tecnológico, hasta aquellos políticos y religiosos.

Además de estas adversidades, también se visualizan otros problemas en la enseñanza del diseńo gráfico. Chaves (2005) subraya que estos provienen de una concepción distorsionada de la profesión, originadas en una disociación entre enseńanza y práctica del oficio, en los cuales predominan dos prejuicios: el teoricismo, que asume al diseńo como teoría aplicada y no como actividad productiva que involucra una serie de habilidades y saberes prácticos siempre cambiantes, restringiéndola a una disciplina racional, desdeñando la realidad y despreciando el trabajo productivo; y el creativismo, postura más universal que adopta el mito de la creatividad vinculada con el arte como matriz y núcleo ontológico del diseño, menospreciando la teoría y la racionalidad.

La integración de ambas perspectivas vendría a ser la relación más conveniente y próxima a la cultura gráfica, algo que Sotelo (2014) enfatiza se está llevando a cabo en la Universidad Nacional Autónoma de México, con el objetivo de promover herramientas para reflexión e impulso de la creación de ideas originales y propositivas en el desarrollo de proyectos, involucrando teoría con el análisis, planeación, innovación y gestión, lo cual lleva implícita la cultura de la interdisciplinariedad y la visión compleja de la realidad. 
Una postura distinta es la de Ariza (2010), quien encuentra que el diseño no ha demostrado ser un área con una evolución similar a su conceptualización al carecer de un aparato crítico reflejándose en poca autoconciencia y autodefinición en su evolución disciplinar, donde sus esfuerzos para generar material teórico se limitan a discursos de tipo analítico, metodologías o sistemas que no pasan de ser especulaciones abstractas e inaplicables a la práctica efectiva.

Se trata de un escenario para formación profesional, en el que la interdisciplinariedad se presenta como elemento transformador, capaz de contribuir a la evolución de la disciplina del diseño, proponiendo un currículo formativo que interrelacione aspectos técnicos, artísticos, teóricos, junto a talleres proyectuales, así como espacios para la discusión que incentiven la reflexión y la crítica, además que conduzcan a la eficiencia e innovación del diseño. Esta postura permite asumir que la formación del diseño gráfico requiere ser enriquecida desde diversos campos del conocimiento.

En síntesis, los programas de diseño gráfico en las universidades de México, como en Latinoamérica, siguen teniendo el reto de flexibilizar en el ejercicio práctico de los planes de estudio, con el fin de lograr un mayor enriquecimiento interdisciplinario entre los programas y la capacidad de adaptación frente a la demanda social que tiene la profesión, aquella que exige la formación de profesionales con capacidades múltiples, poseedores de una visión, y más aún, una cultura compleja, donde la interdisciplinariedad destaca como soporte fundamental; lo cual no implica volver al diseñador experto en múltiples áreas, sino en agente colaborador para la innovación. Para Nae (2017) esto orienta a centrar esfuerzos en preparar a los estudiantes a que trabajen directamente con otras disciplinas. Una labor que recae en los propios diseñadores, al ser los responsables de tomar, implementar y discutir los conocimientos desde otras disciplinas y de establecer puentes con estos (Rodríguez Mendoza, 2016), algo que recién se ha comenzado a reconocer y en pocos casos a aplicar en el currículo como en la misma enseñanza.

A partir de la identificación de las dificultades y retos que enfrenta la enseñanza del diseño gráfico en México, junto a la perspectiva propia que caracteriza al campo profesional, se podrán delinear posibles rutas en busca de una mejor formación del diseñador. De acuerdo con esto, y sustentado en las aportaciones de Brown (2008), Morin (2004), Carvajal (2010), Lyall y Meagher (2012), Fartushenko (2012), Rivera (2013 y 2018), Hayawi y Kamil (2015), Ambrose (2017), en este texto se analiza la pertinencia del diseño gráfico para vincularse con otras disciplinas, desde la perspectiva de los profesores adscritos a los programas de diseño de Instituciones de Educación Superior de México, tanto públicas como privadas. 
Cabe remarcar diferencias entre ambos sectores educativos, dado que mientras la gran mayoría de los investigadores y su productividad, así como una formación de perfil integral tiene lugar en las universidades públicas, en las instituciones privadas dan prioridad a la formación de perfil directivo, en aquellas que tienen gran renombre, o de perfil técnico, en las de menor envergadura. Ambos universos arrojan perspectivas particulares de interés para esta investigación.

\section{Metodología}

Es una investigación de alcance nacional que siguió un enfoque cuantitativo de tipo descriptivo, el cual tuvo como objetivo analizar la pertinencia del grado de uso de la interdisciplina en la formación del diseñador gráfico, de acuerdo con las opiniones de profesores que laboran en diferentes universidades públicas y privadas de México. Con relación a la definición de sujetos, se consideró un muestreo no probabilístico y por conveniencia, el cual fue realizado para obtener respuestas de profesores que estuvieron dispuestos a participar en este estudio.

En cuanto a las características del instrumento utilizado, este fue tipo cuestionario y buscó indagar la percepción de académicos a través de 42 reactivos relacionados con diferentes dimensiones tales como: las estrategias de enseńanza y contenidos curriculares, la perspectiva interdisciplinar en la formación del diseñador, las condiciones académicas, la elección del método de diseño en la enseñanza y la práctica. La definición de este instrumento es un esfuerzo de un grupo de investigación de la Universidad de Sonora (México) que desarrolla el proyecto "Prospectiva del campo profesional del diseño en las IES de México: formación, pensamiento y ejercicio profesional», con el objetivo de analizar la realidad de las áreas del diseño en México y su enseñanza desde las universidades, sustentado en las aportaciones teóricas de Brown (2008), Morin (2004) y otros. Este proyecto ha contado con la participación de otros investigadores del país para concretar, entre otros aspectos, una validación de expertos para el instrumento.

Para la recolección de datos, el estudio realizó la aplicación de una encuesta en formato digital, la cual se logró distribuir a través de correos electrónicos. Como criterio de inclusión, todos los profesores cumplían con desempeñarse en el campo del diseño; de esta forma, se recopiló una muestra de 126 académicos (51\% hombres y $49 \%$ mujeres) que estaban adscritos en diferentes universidades de México como: Universidad Autónoma Metropolitana, Universidad Autónoma de Baja California, Universidad Iberoamericana, 
Universidad Autónoma de Nuevo León, entre otras; el 77\% se desempeñaban en universidades públicas y un $23 \%$ en instituciones privadas. La experiencia académica de los profesores fue de un rango de entre 1 a 46 ańos.

Otras características de la muestra de profesores alcanzada son: $42 \%$ eran de categoría de contratación de tiempo completo (dedicados a desempeñar 40 horas a la semana) y 73 con contrato como profesor de asignatura; $30 \%$ cuentan con estudios de pregrado mientras que $70 \%$ tiene posgrado (maestría o doctorado). En cuanto al nivel de estudios, de los 69 profesores adscritos a universidades públicas, el $85 \%$ cuenta con estudios de posgrado, el resto (14\%) señala solo tener estudios de pregrado. Con relación a una submuestra de sujetos que se desempeñan en universidades privadas, del total de 57 profesores, un $51 \%$ cuentan con posgrado mientras que el $49 \%$ con pregrado. Del total de profesores, solo 11 sujetos de universidades públicas señalaron contar con la distinción del Sistema Nacional de Investigadores.

De esta forma, el diseńo de esta investigación permitió recuperar datos en específico para la conformación de este texto, que se derivaron de indicadores puntuales, referentes a la dimensión de la perspectiva interdisciplinar en la formación del diseñador. También se realizan otros análisis de diferentes niveles de delimitación de la información, haciendo énfasis en encontrar diferencias entre instituciones públicas y privadas, sustentando en las condiciones de los profesores: en las primeras han priorizado una formación integral, estudios de posgrado y actividades de investigación, y en las segundas se ha destacado profesores que provienen del campo profesional, empleados, freelancers, así como directivos. Finalmente se utilizó el grado académico como variable para otras comparaciones y la observación de posibles tendencias en las submuestras. Para el análisis y la delimitación de datos, se utilizó el programa SPSS versión 22 .

\section{Resultados}

Desde la literatura se ubicó cómo el diseño gráfico enfrenta el reto de flexibilizar en el ejercicio práctico de las propuestas formativas a partir de un mayor enriquecimiento interdisciplinario de los programas, incentivando la reflexión y la crítica del estudiante y promoviendo la innovación a través de capacidades múltiples. Andamiaje promotor de capacidades analíticas, diagnósticas y exegéticas como recursos intelectuales. No obstante, en busca de posibles soluciones para aspirar a ese fin, resulta fundamental considerar la perspectiva de los profesores sobre la problemática, al ser estos actores claves en el proceso formativo. 
Los resultados exponen una percepción a favor de la necesidad para el diseño de relacionarse con conocimientos de otras disciplinas, apoyada por el $94 \%$ de los profesores que laboran en universidades públicas, y por el $98 \%$ de aquellos que trabajan en instituciones privadas (ver Figura 1). Cabe destacar que casi un $6 \%$ de quienes trabajan en el sector público le da poca prioridad a la acción interdisciplinar en el diseño, frente a un $2 \%$ de aquellos que se desenvuelven en el sector privado, prevaleciendo un explícito apoyo a la interdisciplinariedad, lo que debe ser valorada como un punto a favor.

Figura 1. Necesidad del diseño en vincularse con conocimientos de otras disciplinas

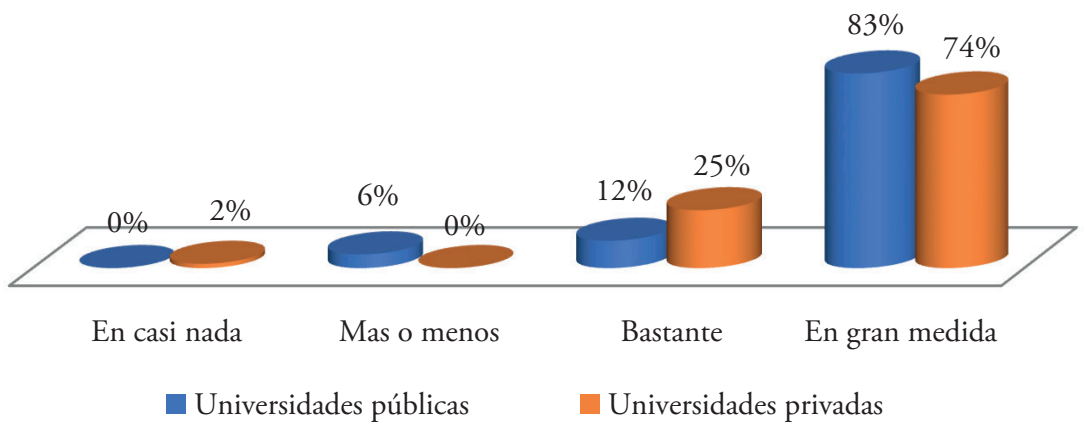

Fuente: Elaboración propia a partir de los datos recopilados.

En relación con la estrategia de enseñanza que el profesor promueve en la formación de sus alumnos, los profesores eligieron entre cuatro estrategias: tradicional (sustentado en el pensamiento lineal-secuencial, heredado de la Bauhaus); interdisciplinar (de perfil sistémico); creatividad pura o creatividad por sí misma (promotor de la creatividad innata de corte artístico como modelo central); y otro (los profesores citaron distintos métodos: analógico natural, constructivista, modelo Ibero, fusión de modelos, modelo propio, lineal-creativo y otros). Categorías que se definieron de acuerdo con las perspectivas de aplicación que sugiere la teoría. Respecto a la estrategia denominada «otros», estas fueron heterogéneas, encontrándose algunas con características lineales, sistémicas como próximas a la creatividad; es decir, algunas con características interdisciplinares como otras alejadas de estas.

Como se puede observar (ver Figura 2), en las instituciones públicas se encuentra un ligero predominio de la interdisciplinariedad en un 54\%, frente a un $30 \%$ del modelo tradicional. Con un $13 \%$ se ubican distintos modelos, y con un $3 \%$ la creatividad pura. 
Figura 2. Estrategia de enseñanza que el profesor promueve en el aula

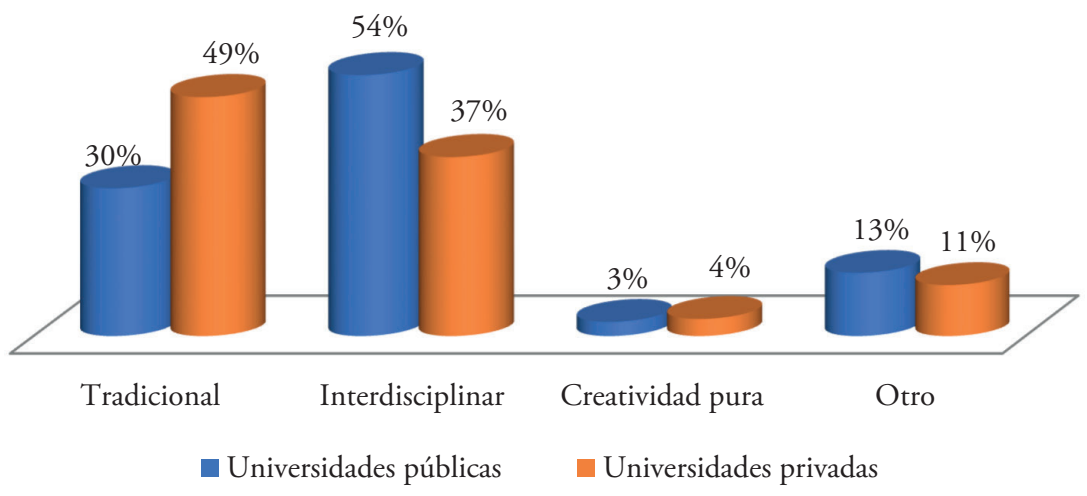

Fuente: Elaboración propia a partir de los datos recopilados.

Porsu parte, los profesores quelaboran en instituciones privadas muestran una tendencia de mayor preferencia (49\%) hacia el modelo tradicional (que replica en la manera de enseñar originado hace un siglo y que ha perdurado en muchos casos como el axioma en la enseñanza del diseño). Del resto de profesores, un $37 \%$ afirma sustentarse en la interdisciplinariedad, $11 \%$ en diversos modelos, mientras que un $4 \%$ confía en la creatividad pura como base de la enseñanza.

El dominio del modelo tradicional presente en las universidades privadas podría ser resultado de la influencia de la visión cartesiana basada en la causalidad (Morín 2004), reflejada luego en el método proyectual y en sí en el pensamiento occidental. Paradigma que ha adoctrinado a las generaciones, como en la Bauhaus, donde se cimentó el andamiaje teórico y enseñanza del diseño. Modelo que contiene los conceptos básicos del diseño pero que conviene ser reevaluado y adecuado en función de los paradigmas sociales, culturales y del diseńo en si del siglo XXI, donde las características de la realidad actual exigen renovadas rutas de pensamiento, no reduccionistas, pero sí incluyentes.

Al respecto Dorochesi (2010) considera que la clave parece estribar en el hecho de no limitarse en la capacidad inventiva individual, sino en que los métodos de trabajo individuales y corporativos que evolucionan como los productos, lo que permite entender que existe un potencial de mejora y evolución en este plano. En consecuencia, esto parece tener mayores posibilidades de ocurrir en las universidades públicas, donde la apuesta por una formación de un perfil integral se perfila más probable y donde algunas instituciones ya están dedicando ciertos esfuerzos. 
Figura 3. Método de diseño que el profesor utiliza al diseñar

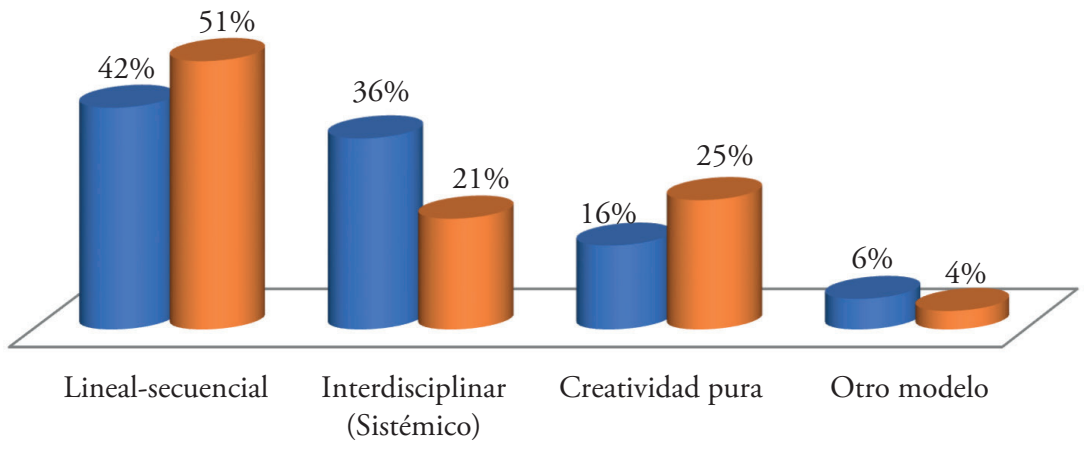

Universidades públicas = Universidades privadas

Fuente: Elaboración propia a partir de los datos recopilados.

En la búsqueda de variables que permitieran analizar de mejor forma el problema de propuestas y estrategias que promuevan una mejor formación de los diseñadores, se consultó el método de diseño que el profesor utiliza o ha utilizado en algún momento de la práctica profesional. Cabe destacar que casi un $30 \%$ de los profesores encuestados se encuentran laborando dentro del giro del diseño, ya sea como empleado, freelance, e incluso dirigiendo empresas.

Paradójicamente, mientras el $96 \%$ de profesores consideraron como necesario para el diseńo relacionarse con los conocimientos de otras disciplinas, propio de una visión compleja y una conducta interdisciplinar, al opinar sobre el método de diseño que ellos utilizan al diseñar (ver Figura 3), no hubo correlación. De aquellos que laboran en universidades públicas, el $42 \%$ señaló aplicar el método tradicional (secuencial-lineal); un 16\% se apoya en el instinto creativo, y un 6\% en otros modelos. En contraparte solo el 36\% afirmó valerse de métodos interdisciplinares en la práctica, a pesar de juzgarlo necesario para la dinámica del diseño.

Con relación a quienes laboran en instituciones privadas, existe mayor discrepancia aún, donde el 51\% aplica el método tradicional; un 25\% la creatividad pura, y el 4\% otros modelos; mientras los métodos de perfil interdisciplinar son considerados por apenas el $21 \%$. En este grupo, solo la quinta parte emplea la interdisciplinariedad en la práctica profesional.

Este último dato resulta relevante, ya que son los profesores que pertenecen a las instituciones privadas quienes en una mayor proporción se desenvuelven en el campo profesional, reflejando un panorama más próximo 
sobre la situación en México, lo cual revela dos realidades distintas: por un lado el 96\% del total de los profesores encuestados asume necesario para el diseńo relacionarse con los conocimientos de otras disciplinas, pero solo el $28.5 \%$ lo lleva a la práctica al diseñar.

Figura 4. Método de diseño que considera es el más utilizado en la práctica profesional

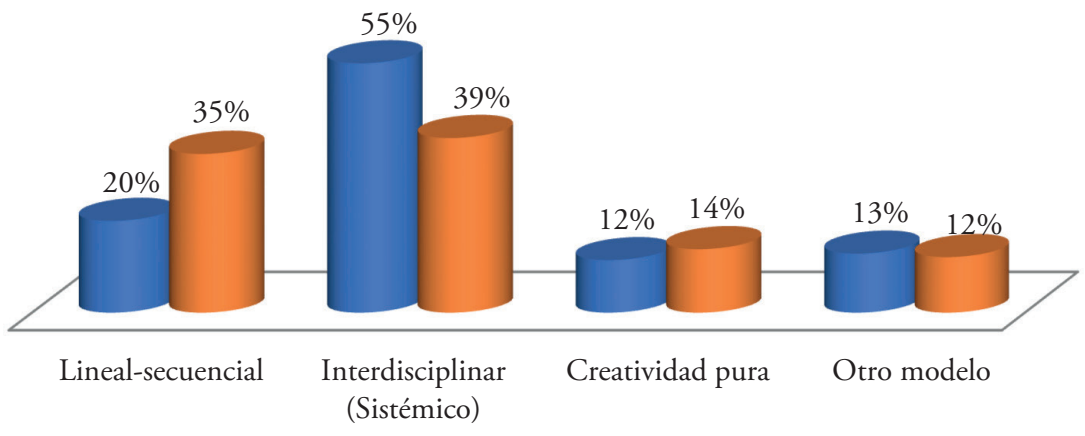

- Universidades públicas Universidades privadas

Fuente: Elaboración propia a partir de los datos recopilados.

En lo referente a la percepción del profesorado, con relación al método de diseño que se aplica en el ejercicio profesional (ver Figura 4), de los profesores que laboran en universidades públicas, un $55 \%$ considera que el método interdisciplinar es el empleado; $20 \%$ el método tradicional; $12 \%$ la creatividad pura, y $13 \%$ otros modelos.

En cuanto a aquellos adscritos a instituciones privadas, en proporciones muy similares que promedian 37\%, conciben que prácticamente a la par se emplean los modelos tradicional e interdisciplinar, y en un nivel menor que ronda $13 \%$, ubican la creatividad y otros modelos.

Estas perspectivas dejan ver dos criterios distintos; uno de ellos se refiere a que los profesores que laboran en instituciones privadas detectan en mayor presencia el modelo tradicional lineal, muchos de los cuales se encuentran activos en el campo laboral, lo que probablemente sea un reflejo de lo que acontece en la actividad profesional. En contraparte, aquellos que pertenecen a las universidades públicas y quienes tienden a conocer de mejor manera los diversos métodos, también por tener en mayor proporción estudios de posgrado y acceso a la investigación, aunque menos presencia en el campo laboral, perciben mayor presencia de modelos interdisciplinares. 
De esto emerge un tercer hallazgo: a mayor grado académico (85\% de profesores adscritos a universidades públicas) hay mayor tendencia por la interdisciplinariedad, mientras a menor grado académico $(51 \%$ de profesores empleados a universidades privadas) se opta por los métodos tradicionales.

\section{DisCUSIÓN Y CONCLUSIONES}

Desde la perspectiva tradicional, el diseñador gráfico se caracteriza por recibir una formación a través de la cual cumple tareas que, de forma creativa, le permite resolver problemas. En el contexto de la actual sociedad, el diseño ha experimentado una complejidad y dinámica evidente, en la que sus necesidades trascienden a los problemas establecidos, siendo necesario asimilar información poco clara desde fuentes heterogéneas, así como anticiparse a los nuevos problemas, incluso a aquellos próximos a emerger; lo que hace del diseño una especialización que per se, requiere de una formación que trascienda los confines de la misma disciplina, que enriquezca los recursos intelectuales y promueva el análisis, la crítica y la innovación del diseñador. Esto implica abordar los problemas y su contexto desde perspectivas más amplias, donde se asume a la interdisciplinariedad como posible ruta a través de la cual hacer frente a las situaciones cuya complejidad rebasa los límites disciplinares y que son cada vez más comunes. Coyuntura que no se percibe en la formación como en el currículo de los programas de diseño gráfico.

Lo anterior se puede asumir teórica y empíricamente, desde donde emergen dos realidades: por un lado, prácticamente el total de profesores encuestados percibe que el diseño no puede seguir concibiéndose desde los mismos paradigmas que han sustentado su teoría y práctica, originados en el siglo XX, dadas las particularidades contextuales del siglo XXI, percibiéndose a la interdisciplinariedad como herramienta necesaria y favorable para ese fin. Sin embargo, en cuanto estrategia de enseñanza que el docente promueve en el aula, la interdisciplinariedad es considerada solo por poco más del 50\% de aquellos que laboran en universidades públicas, y apenas por el $37 \%$ de quienes pertenecen a instituciones privadas, lo que exhibe discrepancia entre lo que los profesores perciben respecto a la disciplina exógenamente y su labor endógena. A esto se suma que los planes de estudios de la mayoría de las universidades en México no incorporan la perspectiva interdisciplinar, y si lo hacen, es más en forma circunscrita.

Siguiendo esta tendencia, en cuanto al método que los profesores utilizan al diseñar, estos emplean de manera limitada los modelos interdisciplinares: apenas un 36\% de quienes laboran en universidades públicas y $21 \%$ de quienes 
lo hacen en instituciones privadas. Semejante es la opinión respecto al método de diseño que considera el profesor es el más utilizado por los diseñadores en la práctica profesional y responsable de establecer su forma de trabajar, donde los interdisciplinares para quienes laboran en instituciones privadas ascienden a un 39\%, mientras para aquellos adscritos a universidades públicas es un $55 \%$. Igualmente se manifiesta una tendencia: los profesores con posgrado tienden a la interdisciplinariedad en mayor proporción (85\%), frente a aquellos con pregrado (51\%), siendo el grado académico un factor importante en este sentido.

Pese a las limitaciones metodológicas, principalmente por el tipo de muestreo, ya que participa un número parcial de profesores adscritos a universidades públicas y un número inferior de quienes laboran en instituciones privadas, siendo el tipo de sujeto que se indaga así como la ubicación geográfica factores importantes; existen conclusiones relevantes a destacar: se focaliza que los profesores casi en su totalidad perciben que la epistemología, teoría, metodología y práctica del diseño, requieren ampliarse y actualizarse a través de nuevas vías, considerando a la interdisciplinariedad como una de ellas.

Esto también tiene efectos en la discusión sobre la cuestión curricular del diseño en México, la cual ha sido enfocada en la propuesta de una enseñanza con base en las competencias profesionales (Sánchez y Aguilera, 2010), lo cual alimenta un tipo de enseñanza que también tiene dependencia de los intereses de cada institución por responder a las necesidades sociales y al mercado laboral de contexto propio. Esto a su vez, reconoce un alejamiento de los modelos de enseñanza del diseño idea (Oleas, Padilla y Cayambe, 2018) que postulan un equilibrio entre el arte, la ciencia y tecnología, los cuales se desprenden de aquellos primeros diseńos pedagógicos propuestos por la Bauhaus que son continuados por la Escuela de Ulm.

Entonces, más si se trata de una visión holística e integral de lo interdisciplinar, concebida prioritariamente como una estrategia pedagógica que conceptualice los propósitos y la planificación del proceso (Carvajal, 2010), es pertinente comprender que transferir esto a la realidad del diseño, deja de ser una tarea sencilla, dado que se requiere un compromiso y acción conjunta que involucra universidades y profesores, el entorno empresarial, así como a la misma comunidad de diseńadores con una tendencia a flexibilizar y ampliar el marco de referencia de la realidad. Una tarea que a pesar de las dificultades que presenta comienza a tener lugar, particularmente en el escenario universitario. En este sentido, Pimentel (2010) explica que la formación de los futuros diseñadores implica aprender a pensar en contextos más amplios de relaciones, donde el futuro de la disciplina parece apuntar a investigar, interpretar 
y proponer modelos de enseñanza que promuevan la confrontación creativa e innovadora del diseño con la racionalidad industrial y económica.

Coincidiendo con Rodríguez y Montiel (2020), el saber y hacer del diseñador hoy implica potencialmente aprender a ser creativo, innovador e ingenioso, sustentado en una inteligencia múltiple, más allá de solo la intuición o creatividad innata, lo que involucra la práctica interdisciplinar. Coyuntura que da forma a la realidad presente del diseño gráfico, donde la interdisciplinariedad como pilar inherente contribuye en la conformación de una nueva cultura del diseño. Paradigma que además requeriría de un soporte formativo comprometido en capacitar a los estudiantes en el análisis, razonamiento y en general, dentro del desarrollo de herramientas cognitivas adecuadas para las nuevas necesidades sociales, culturales y del mercado, y que mutan constantemente. Escenario que demanda docentes con amplio dominio de su disciplina y actualización constante de acuerdo con las tendencias y necesidades laborales y profesionales.

Esto podría llevar al diseñador de su actual perfil reproductor al progresista; dejando atrás, como sentencia Dorochesi (2010), conceptos de carácter más egocéntrico que por décadas han dejado al diseño en Latinoamérica como un fenómeno de características más bien académicas que empresariales; para lo cual Tresserras (2015) propone reconsiderar los planteamientos pedagógicos ante el mayor protagonismo de la interdisciplinariedad, redefiniendo los planes de estudios guiados por asignaturas de talleres como eje central, que incluyan proyectos caracterizados por una complejidad creciente, entretejidos con el resto de las asignaturas de manera sinérgica; pues de poco servirá dichos talleres si no incentivan el desarrollo de herramientas cognitivas (Rivera, 2018).

En el caso de las propuestas formativas del diseño gráfico en las universidades de México, se identifican necesidades en la acentuación de la formación orientada hacia tres áreas principales:

1. El enriquecimiento de los recursos intelectuales, lógico-reflexivos, lo cual es resultado de la cantidad escasa de asignaturas detonadoras de estas habilidades, así como también de espacios que propicien el diálogo y la discusión. Por tanto, se propone el incremento de asignaturas teóricas que sean dinámicas, donde se incentiven la reflexión y la creatividad intelectual en el diseño y más allá de este.

2. Necesidad de un mayor conocimiento de los modelos metodológicos para la creación gráfica, pues tal como establecen Leonor, Chaves y Ledesma (1997), la metodología en el diseño no se limita al discurso sobre aplicaciones mecánicas y simplistas de esquemas operativos 
donde predomina más el modelo que el caso, sino de nuevas modalidades de aplicación del discurso teórico a las disciplinas proyectuales donde la reflexión sobre el procedimiento es tan importante como el proceso mismo. En consecuencia, los alcances y ventajas de la reflexión de modelos como el interdisciplinar, incentivaría en el estudiante la comprensión y definición de propuestas de soluciones integrales sobre los problemas de diseño que enfrenta.

3. Necesaria formación en el área de gestión, limitada por el poco espacio de asignaturas con dicho enfoque en la mayoría de las propuestas de formación, así como la proporción escasa de profesores con experiencia en el ámbito profesional; lo que se traduce en una vinculación deficiente con el sector productivo empresarial. Para ello se propone formar al estudiante a través del desarrollo de proyectos que promuevan un ambiente similar al que se vive en el campo laboral, impulsando el análisis, creatividad e innovación a través del desarrollo de actividades generadas vinculando a los estudiantes en proyectos con la iniciativa privada.

Si tal propósito se asume, requeriría del desarrollo de propuestas formativas multidisciplinares donde además las asignaturas se diseñen enfocadas en una forma sistémica, capaces de interrelacionar conocimientos teóricos, técnicos, metodológicos, proyectuales y creativos, incentivados por el diálogo como promotor de la reflexión, alejándose de formar grupos de conocimientos aislados, lo cual favorecerá a la formación de capacidades necesarias en el diseñador del siglo XXI.

\section{REFERENCIAS BIBLIOGRÁFICAS}

Ambrose, D. (2017). Large-Scale Interdisciplinary design thinking for dealing with twenty-first century problems and opportunities En F. Darbellay, Z, Moody y T. Lubart (eds.), Creativity, Design Thinking and Interdisciplinarity (pp. 35-52). Singapur: Springer Singapore. https://doi. org/10.1007/978-981-10-7524-7_3

Ariza, S. (2010). Elementos característicos de los programas diseño. Actas de Diseño, 9(5), 49-55. Recuperado de https://fido.palermo.edu/servicios_ dyc/publicacionesdc/archivos/148_libro.pdf

Brandão, A. L., García, M. C., Lozano, D. E., Llano, S. E. P., de Oliveira, L. P. M., Pinto, A. J., ... y Marín, Z. S. (2014). Interdisciplina: la enseñanza/aprendizaje en proyectos de diseño urbano. On the w@terfront, (29), 22-40.

Brown, T. (2008). Design thinking. Harvard Business Review, 86(6), 84-92. 
Calvache, D. S. (2017). La metodología de diseño al interior de los programas de diseño industrial colombiano: hacia el planteamiento de formas propias de hacer diseño. Actas de Diseño, (24), 218-221.

Carvajal, Y. (2010). Interdisciplinariedad: desafío para la educación superior y la investigación. Revista Luna Azul, (31),156-169.

Cepeda, M. J. y Carrillo, J. C. S. (2019). La formación de profesionales en el diseño gráfico. Estudio de las competencias y habilidades necesarias para el campo laboral. Actas de Diseño, 26(13), 133-139.

Chaves, N. (2005). Dos distorsiones en la enseñanza del diseño gráfico. Foro Alfa. Recuperado de https://foroalfa.org/articulos/dos-distorsiones-en-laensenanza-del-diseno-grafico

Chou, W. H. y Wong, J. J. (2015). From a Disciplinary to an Interdisciplinary Design Research: Developing an Integrative Approach for Design. International Journal of Art and Design Education, 34(2), 206-223. https:// doi.org/10.1111/jade.12017

Donoso Cisternas, S. (2016). El Diseño Industrial; las fronteras confusas de la creatividad. Ciencia, docencia y tecnología, 27(53), 342-361.

Dorochesi Fernandois, M. R. (2010). Complejidad, creatividad y cambio. Reflexiones en torno a los nuevos escenarios para la enseñanza del Diseño. Actas de Diseño, 9(5), 95-101. Recuperado de https://fido.palermo.edu/ servicios_dyc/publicacionesdc/archivos/148_libro.pdf

D'souza, N. (2016). Investigating design thinking of a complex multidisciplinary design team in a new media context: Introduction. Design Studies, (46), 1-5. https://doi.org/10.1016/j.destud.2016.07.001

Fartushenko, L. (2012). An interdisciplinary approach to promote creativity. Designedasia, 3(1), 1-8.

García-Cordero, M. S. y Buitrago-López, Y. C. (2017). Modelo pedagógico de pensamiento complejo en diseño gráfico. Revista Logos, Ciencia \& Tecnologia, 8(2), 117-131. https://doi.org/10.22335/rlct.v8i2.392

Hayawi, A. y Kamil, L. (2015). Graphic Design as Means of Student's Creativity Activity. International Journal of Social Science and Humanity, 5(3), 248-251. https://doi.org/10.7763/IJSSH.2015.V5.462

Herrera, L. y Neve, M. (noviembre, 2011). Laboratorio de diseño en el currículo de diseńo gráfico sustentado en el aprendizaje situado y la metacognición. Memorias del XI Congreso Nacional de Investigación Educativa. Ciudad de México, México.

Kirci, N. (2016). The transfer of knowledge through inter-disciplinary seminars as a source of creativity in the design studio. AD ALTA-Journal of Interdisciplinary Research, 6(1), 26-31. 
Leonor, A., Chaves, N. y Ledesma, M. (1997). Diseño y comunicación. Teorías y enfoques críticos. Buenos Aires: Paidós.

Lyall, C. y Meagher, L. (2012). A Masterclass in interdisciplinarity: Research into practice in training the next generation of interdisciplinary researchers. Futures, 44(6), 608-617. https://doi.org/10.1016/j.futures.2012.03.011

Morin, E. (2004). Introducción al pensamiento complejo. Barcelona: Gedisa.

Mose Biskjaer, M., Dalsgaard, P. y Halskov, K (2017). Understanding creativity methods in design. Proceedings of the 2017 conference on designing interactive systems, 839-851. New York: Asociation for Computing Machinery. https://doi.org/10.1145/3064663.3064692

Nae, H. (2017). An interdisciplinary design education framework. The Design Journal, 20(1), 835-847. https://doi.org/10.1080/14606925.2017.1353030

Oleas, J., Padilla, N. y Cayambe, Á. (2018). Modelos de Enseñanza del Diseño: Influencia de Bauhaus y HfG de Ulm. Revista Cientifica Investigar, 7, 39-53.

Pimentel Jiménez, J. V. (2010). Formando diseñadores de interiores del Siglo XXI con planes de estudio del Siglo XX. Actas de Diseño, 9(5), 223-226. Recuperado de https://fido.palermo.edu/servicios_dyc/publicacionesdc/ archivos/148_libro.pdf

Rivera, E., Higuera, A., Rubio, M., Utrilla, S. y Santamaría, A. (2014). La educación holística para los profesionales del diseño. Revista Iberoamericana de Producción Académica y Gestión Educativa, 1(1), 1-21. Recuperado de https://www.pag.org.mx/index.php/PAG/article/view/85

Rivera, L. A. (2013). La Nueva Educación del Diseño Gráfico. Ciudad de México: Editorial Designio.

Rivera, L. A. (2018). La evaluación de la educación del diseño en México: un enfoque desde la didáctica. Ciudad de México: Comaprod.

Rodríguez Mendoza, R. M. (2016). La pedagogía del diseño gráfico basada en la investigación en diseño. Revisión bibliográfica. Iconofacto, 12(19), 254-267. https://doi.org/10.18566/iconofact.v12.n19.a11

Rodríguez, I. y Montiel, O. (2020). Diseńo y Emprendimiento: su enseñanza y complejidad en una universidad pública. Actas de Diseño, 15(32), 214-218.

Roncallo-Dow, S. (2013). Entre la interdisciplinariedad, las epistemologías y los objetos. Palabra Clave, 16(2), 269-281.

Sánchez, M. (2016). La conceptualización del diseño. Actas de Diseño, (20), 237-240. Recuperado de http://fido.palermo.edu/servicios_dyc/publicacionesdc/archivos/588_libro.pdf

Sánchez, M. y Aguilera, J. (2010). La enseñanza del diseño gráfico en base a las competencias profesionales. Actas de Diseño, 8(4). 
Sotelo Leal, S. (2014). Ensayo crítico sobre la enseñanza del diseño gráfico en México. Encuadre, Asociación Mexicana de Escuelas de Diseño Gráfico. Recuperado de https://encuadre.org/ensayo-critico-sobre-la-ensenanza-deldiseno-grafico-en-mexico/

Soto, R, (1993). Propuesta para un modelo curricular flexible. Revista de la Dducación Superior, 22(85), 1-9. Recuperado de http://publicaciones. anuies.mx/pdfs/revista/Revista85_S2A4ES.pdf

Tresserras, J. (2015). Diseño e interdisciplinariedad. Una visión. On the W@terfront. Public Art. Urban Design. Civic Participation. Urban Regeneration, 34(2), 5-18.

Vázquez-Alonso, Á. y Manassero-Mas, M. A. (2017). Interdisciplinariedad y conceptos nómadas en didáctica de la ciencia: consecuencias para la investigación. Revista Eureka sobre Enseñanza y Divulgación de las Ciencias, 14(1), 24-37. https://doi.org/10.25267/Rev_Eureka_ensen_divulg_cienc.2017. v14.i1.03 